\title{
$\Lambda$ hyperons and the neutron drip line
}

\author{
Xian-Rong Zhou (周先荣), ${ }^{1}$ A. Polls, ${ }^{2}$ H.-J. Schulze,${ }^{3}$ and I. Vidaña ${ }^{2}$ \\ ${ }^{1}$ Department of Physics and Institute of Theoretical Physics and Astrophysics, Xiamen University, Xiamen 361005, People's Republic of China \\ ${ }^{2}$ Departament d'Estructura i Constituents de la Matèria, Universitat de Barcelona, E-08028 Barcelona, Spain \\ ${ }^{3}$ INFN Sezione di Catania, Via Santa Sofia 64, I-95123 Catania, Italy
}

(Received 31 July 2008; published 7 November 2008)

\begin{abstract}
We study the influence of $\Lambda$ hyperons on neutron drip properties in hypernuclear matter and hypernuclei, using a microscopic $\Lambda N$ force. We find an extension of the neutron drip line in bulk matter and stabilization of neutron-rich isotopes due to added hyperons.
\end{abstract}

DOI: 10.1103/PhysRevC.78.054306

PACS number(s): 21.80.+a, 21.60.Jz, 21.65.-f, 21.10.Dr

\section{INTRODUCTION}

New experimental facilities under construction at GSI, JLAB, J-PARC, and other sites will soon allow a much more precise determination of the properties of hyperon-nucleon and hyperon-hyperon forces than is currently available (see Ref. [1] for a recent account of experimental data). Initial investigations of hypernuclear physics were mainly focused on the spectroscopy of single- $\Lambda$ hypernuclei. The quantitative information obtained and its theoretical analysis became one of the most relevant tools to constrain $\Lambda N$ interactions. Complementary to these studies, in this article we consider a particular feature of hypernuclear physics that might be accessible in the future [2]: The change of nuclear structure due to the effect of added hyperons. In particular we focus on the modification of states close to the neutron drip line when adding one or more $\Lambda$ hyperons to the system. A detailed study of this effect in infinite hypernuclear matter will be complemented by the consideration of some typical hypernuclei.

This subject has generated some theoretical interest in the past and, apart from the exploration of hypernuclear bulk matter [3-6], several studies of neutron-rich hypernuclei have been performed in different theoretical frameworks. We mention the relativistic mean-field treatments of Refs. [7] and [8], the Skyrme-Hartree-Fock approach of Ref. [9], and the use of a generalized mass formula in [10].

Obviously the results depend, apart from the theoretical scheme, on the nucleon-nucleon and hyperon-nucleon interactions that are used. The purpose of our study is to employ a microscopically derived hyperon-nucleon force together with recent reliable nucleonic interactions suitable for neutron-rich environments. More precisely, we use for this purpose a microscopic in-medium $\Lambda N$ force without adjustable parameters, derived from Brueckner-Hartree-Fock (BHF) calculations of isospin-asymmetric hypernuclear matter $[5,11]$ with the Nijmegen soft-core hyperon-nucleon potential NSC89 [12] and the Argonne $V_{18}$ nucleon-nucleon interaction [13], including explicitly the coupling of the $\Lambda N$ to the $\Sigma N$ states. This $\Lambda N$ force is combined with a standard Skyrme force for the nucleon-nucleon interaction to calculate the properties of homogeneous hypernuclear matter, while hypernuclei are treated in a Skyrme-Hartree-Fock (SHF) model employing the same interactions and including quadrupole deformations and (nucleonic) pairing. This methodology gives access to more refined information than just using a generalized mass formula [10]. Furthermore, the microscopically founded $\Lambda N$ interaction that we use avoids the uncertainties of the parametrizations of the $\Lambda N$ Skyrme forces used in Ref. [9].

In the next section, we briefly review the necessary formalism. The results for hypernuclear matter and hypernuclei are presented in Sec. III and the main conclusions are summarized in the last section.

\section{FORMALISM}

For the nucleonic energy density functional $\varepsilon_{N}$ to be used for infinite hypermatter or finite nuclei we choose a standard Skyrme functional with the modern Skyrme forces SkI4 [14] or SLy4 [15], which have been specifically devised with attention to the description of neutron-rich systems. For the calculations in the homogeneous system, we also consider alternatively a simple analytical energy density functional developed in Ref. [16] (hereafter referred to as Av18+3BF), which parametrizes the results of the variational calculation in the framework of correlated basis functions with Argonne $V_{18}$ potential plus a Urbana three-body force and relativistic boost corrections of Ref. [17]. These variational results are in very good agreement with BHF calculations [16]. This energy density functional is expressed in terms of a compressional and a symmetry term:

$$
\begin{aligned}
\varepsilon_{N} & =\varepsilon_{0}\left(\rho_{N}\right)+\varepsilon_{\mathrm{sym}}\left(\rho_{N}\right) \alpha^{2} \\
& =\rho_{N}\left(E_{0} u \frac{u-2-\delta}{1+u \delta}+S_{0} u^{\gamma} \alpha^{2}\right) .
\end{aligned}
$$

In this expression, $\rho_{N}=\rho_{n}+\rho_{p}$ is the total nucleonic density, $\alpha=\left(\rho_{n}-\rho_{p}\right) / \rho_{N}$ the nucleon asymmetry, and $u=\rho_{N} / \rho_{0}$ the ratio of the nucleonic density to nuclear saturation density. The best fit of the variational calculations of Ref. [17] with this simple functional is obtained with $\rho_{0}=0.16 \mathrm{fm}^{-3}, E_{0}=$ $15.8 \mathrm{MeV}, S_{0}=32 \mathrm{MeV}, \gamma=0.6$, and $\delta=0.2$.

The contribution to the energy density functional due to the presence of hyperons, $\varepsilon_{\Lambda}$, is written as [18]

$$
\begin{aligned}
\varepsilon_{\Lambda}= & \frac{\tau_{\Lambda}}{2 m_{\Lambda}}+\varepsilon_{N \Lambda}\left(\rho_{n}, \rho_{p}, \rho_{\Lambda}\right) \\
& +\left(\frac{m_{\Lambda}}{m_{\Lambda}^{*}\left(\rho_{n}, \rho_{p}, \rho_{\Lambda}\right)}-1\right) \frac{\tau_{\Lambda}-C \rho_{\Lambda} 5 / 3}{2 m_{\Lambda}}
\end{aligned}
$$


with $\tau_{\Lambda} / 2 m_{\Lambda}$ being the $\Lambda$ kinetic energy density, $C=$ $\left(3 \pi^{2}\right)^{2 / 3} 3 / 5 \approx 5.742$, and

$$
\begin{aligned}
\varepsilon_{N \Lambda}= & \left(\rho_{n}+\rho_{p}+\rho_{\Lambda}\right) \frac{B}{A}\left(\rho_{n}, \rho_{p}, \rho_{\Lambda}\right) \\
& -\left(\rho_{n}+\rho_{p}\right) \frac{B}{A}\left(\rho_{n}, \rho_{p}, 0\right)-\frac{C \rho_{\Lambda}{ }^{5 / 3}}{2 m_{\Lambda}} .
\end{aligned}
$$

The last term in Eq. (2) vanishes in homogeneous hypermatter. These energy functionals are obtained from a fit to the binding energy per baryon, $B / A\left(\rho_{n}, \rho_{p}, \rho_{\Lambda}\right)$, of asymmetric hypermatter, as generated by $\mathrm{BHF}$ calculations $[5,11]$. The adequate $\Lambda$ effective mass (used also in the SHF Schrödinger equation),

$$
\frac{m_{\Lambda}^{*}}{m_{\Lambda}}=\left(1+\frac{U_{\Lambda}\left(k_{F}^{(\Lambda)}\right)-U_{\Lambda}(0)}{k_{F}^{(\Lambda)^{2}} / 2 m_{\Lambda}}\right)^{-1}
$$

is computed from the BHF single-particle potentials $U_{\Lambda}(k)$ obtained in the same calculations. In practice we use the following parametrizations of energy density and $\Lambda$ effective mass in terms of the partial densities $\rho_{n}, \rho_{p}, \rho_{\Lambda}\left(\rho_{N}\right.$ and $\rho_{\Lambda}$ given in units of $\mathrm{fm}^{-3}, \varepsilon_{N \Lambda}$ in $\mathrm{MeV} \mathrm{fm}^{-3}$ ):

$$
\begin{aligned}
\varepsilon_{N \Lambda} \approx & -\left[368-\left(1717+268 \alpha-920 \alpha^{2}\right) \rho_{N}\right. \\
& \left.+\left(2932-776 \alpha+2483 \alpha^{2}\right) \rho_{N}^{2}\right] \rho_{N} \rho_{\Lambda} \\
& +\left[449-2470 \rho_{N}+5834 \rho_{N}^{2}\right] \rho_{N} \rho_{\Lambda}{ }^{5 / 3} \\
\frac{m_{\Lambda}^{*}}{m_{\Lambda}} \approx & 1-\left[1.58+0.12 \alpha-0.12 \alpha^{2}+0.54 y-0.14 y^{2}\right] \rho_{N} \\
+ & +\left[4.11+2.11 \alpha+2.88 \alpha^{2}+0.35 y+1.17 y^{2}\right] \rho_{N}{ }^{2} \\
& -\left[4.03+7.08 \alpha+5.18 \alpha^{2}-0.93 y+3.27 y^{2}\right] \rho_{N}{ }^{3}
\end{aligned}
$$

where $y=\rho_{\Lambda} / \rho_{N}$.

From the total energy density of hypernuclear matter, $\varepsilon=$ $\varepsilon_{N}+\varepsilon_{\Lambda}$, one can then calculate the chemical potentials

$$
\mu_{i}=\frac{\partial \varepsilon}{\partial \rho_{i}}, \quad i=n, p, \Lambda,
$$

and the pressure of the system,

$$
p=\rho^{2} \frac{\partial}{\partial \rho} \frac{\varepsilon}{\rho}, \quad \rho=\rho_{N}+\rho_{\Lambda} .
$$

Regarding the description of hypernuclei, we use the SHF formalism developed in Refs. [18-20], using the $\Lambda N$ energy density functional Eq. (2) together with the same nucleonic Skyrme force as in infinite hypermatter. This formalism reproduces fairly well the binding energies and single-particle levels of $\Lambda$ hypernuclei and can thus be considered sufficiently reliable for our purpose. Microscopic calculations of the $\Lambda$ self-energy for finite nuclei in certain simple cases (closed shells) and using realistic $N N$ [13] and $Y N$ [12] interactions are also available and provide good agreement with the single-particle levels of $\Lambda$ hypernuclei [21].

In Ref. [20], the energy density functional has been extended to arbitrary nuclear asymmetry, since we are now interested in very neutron-rich nuclei. Furthermore, modern nucleonic Skyrme forces (SkI4 [14] or SLy4 [15]) suitable for this situation are now used and we include, as already said, the effects of quadrupole deformation and (nucleonic) pairing.

\section{RESULTS}

\section{A. Hypernuclear matter}

We are mainly interested in the neutron drip properties, determined by the vanishing of the neutron chemical potential, of asymmetric hypermatter characterized by the total baryonic density $\rho_{N}$, the nucleon asymmetry $\alpha$, and the presence of a certain amount of $\Lambda$ 's described by $\rho_{\Lambda}$. We consider the case of saturated hypermatter with vanishing pressure as it is the most significant situation for the analysis of finite nuclei.

Figure 1 shows the different chemical potentials, $\mu_{n}$ and $\mu_{p}$ in the left panel and $\mu_{\Lambda}$ in the right panel, as a function of nucleon asymmetry for different fixed $\Lambda$ densities $\left(\rho_{\Lambda}=0.0,0.02,0.04 \mathrm{fm}^{-3}\right)$ under the condition of vanishing pressure, obtained with the SLy4 force together with the parametrization of Eq. (5) to describe the nucleonic and the hyperonic contributions, respectively. As expected, the proton chemical potential decreases with the nuclear asymmetry, while the neutron chemical potential increases, and the crossing with zero defines the maximum (neutron drip) asymmetry for a given value of $\rho_{\Lambda}$. The neutron drip asymmetry increases with the presence of $\Lambda$ 's, which act as an additional source of attraction for neutrons. One thus expects that for finite nuclei the presence of one or more $\Lambda$ 's should translate in an increment of the number of neutrons that a nucleus can support.

When the nucleon asymmetry vanishes, $\mu_{n}$ and $\mu_{p}$ coincide if $\rho_{\Lambda}=0$. However, the presence of $\rho_{\Lambda}$ produces an isospin breaking asymmetry (induced by the underlying NSC89 potential [12]) and the chemical potentials of neutrons and protons become different even at $\alpha=0, \mu_{p}$ being slightly
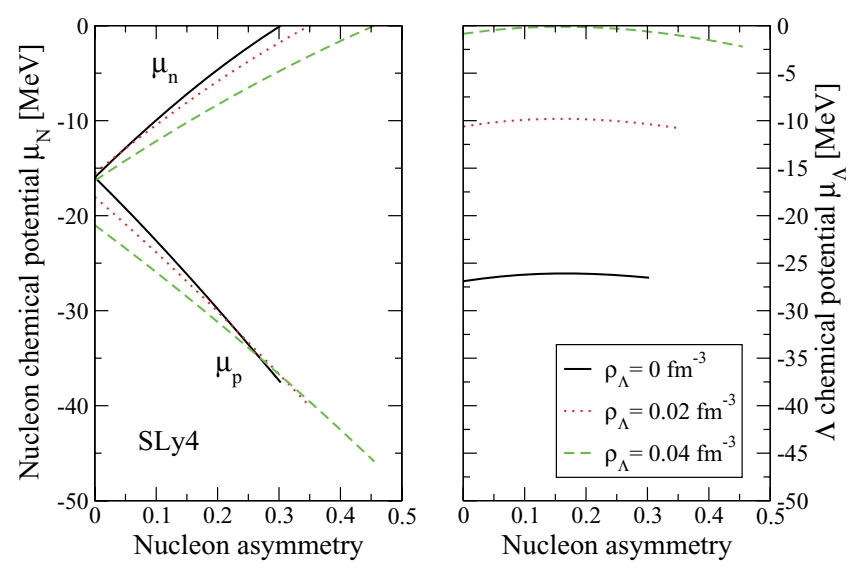

FIG. 1. (Color online) Neutron, proton, and lambda chemical potentials at zero pressure as a function of the nucleon asymmetry for different values of $\rho_{\Lambda}$, obtained with the SLy4 interaction together with the parametrization of Eq. (5). $\mu_{n}$ and $\mu_{p}$ are shown in the left panel, while $\mu_{\Lambda}$ is plotted in the right panel. The solid, dotted, and dashed lines correspond to $\rho_{\Lambda}=0.0,0.02$, and $0.04 \mathrm{fm}^{-3}$, respectively. 
more attractive than $\mu_{n}$, which is changing very little with $\rho_{\Lambda}$ for low asymmetries.

The $\Lambda$ chemical potential at $\rho_{\Lambda}=0$ (impurity case) has typical values of about $-27 \mathrm{MeV}$ (hyperon well depth), and becomes more repulsive when $\rho_{\Lambda}$ increases, mainly due to the increase of the Fermi motion of the $\Lambda$ 's and the fact that some of the $\Lambda N$ bonds are replaced by $\Lambda \Lambda$ bonds. For all values of $\rho_{\Lambda}$ the dependence of $\mu_{\Lambda}$ on the nucleon asymmetry is very smooth, presenting a shallow maximum at low asymmetries. For $\rho_{\Lambda} \approx 0.04 \mathrm{fm}^{-3}$ the $\Lambda$ chemical potential becomes positive and no more $\Lambda$ 's can be bound by the matter. However, this $\Lambda$ drip point is in practice unreachable, because $\Lambda \Lambda \rightarrow \Xi N$ conversion sets in before $[4,5]$.

One should keep in mind the condition of zero pressure along the curves, such that each asymmetry corresponds to a different total density. In addition, for a given asymmetry, the different values of $\rho_{\Lambda}$ correspond also to different values of the nucleonic density. Figure 2 illustrates this condition by showing the saturation baryon density as a function of nucleon asymmetry for several values of $\rho_{\Lambda}$, obtained with SLy4 for the nucleonic energy density. One observes that the total baryon density at zero pressure decreases with the asymmetry, due to the increment of the Fermi motion and pressure and concurrent reduction of the attractive interaction energy with the asymmetry. Therefore the total density decreases to keep the pressure equal to zero. On the other hand, the increase of the total density with the $\Lambda$ partial density can be understood using the same type of arguments, i.e., the presence of $\Lambda$ 's decreases the Fermi motion and therefore one needs to increase the density in order to keep the pressure constant. For each value of $\rho_{\Lambda}$, the curve is shown up to the nucleon asymmetry corresponding to the neutron drip condition.

As expected, this asymmetry increases with $\rho_{\Lambda}$, as can be seen in Fig. 3, where we show the maximum nucleon asymmetry corresponding to the neutron drip condition, as a function of $\rho_{\Lambda}$ for the three different interactions considered in

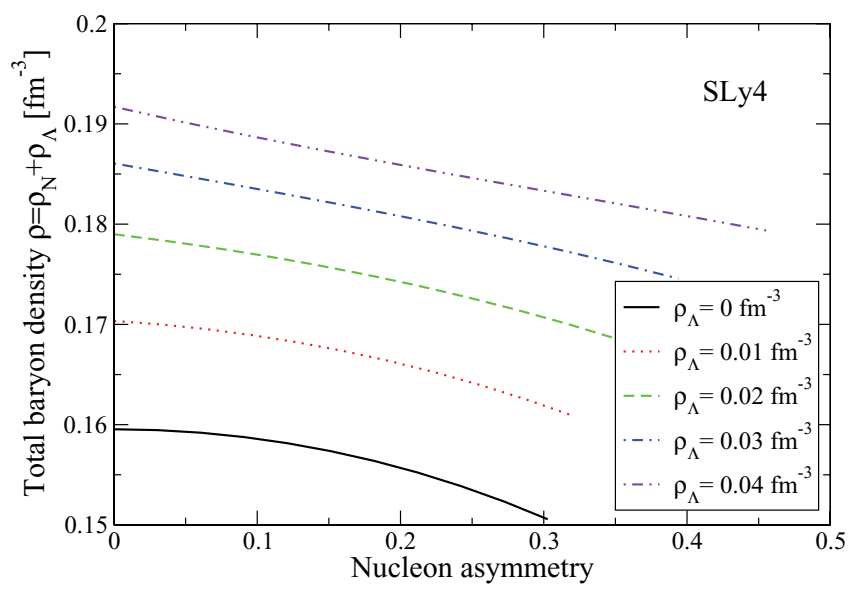

FIG. 2. (Color online) Total baryon density at zero pressure as a function of the nucleon asymmetry for different values of $\rho_{\Lambda}$, obtained with the SLy4 interaction for the nucleonic energy density. Going from low to high densities, the different curves correspond to $\rho_{\Lambda}=$ $0.0,0.01,0.02,0.03,0.04 \mathrm{fm}^{-3}$.

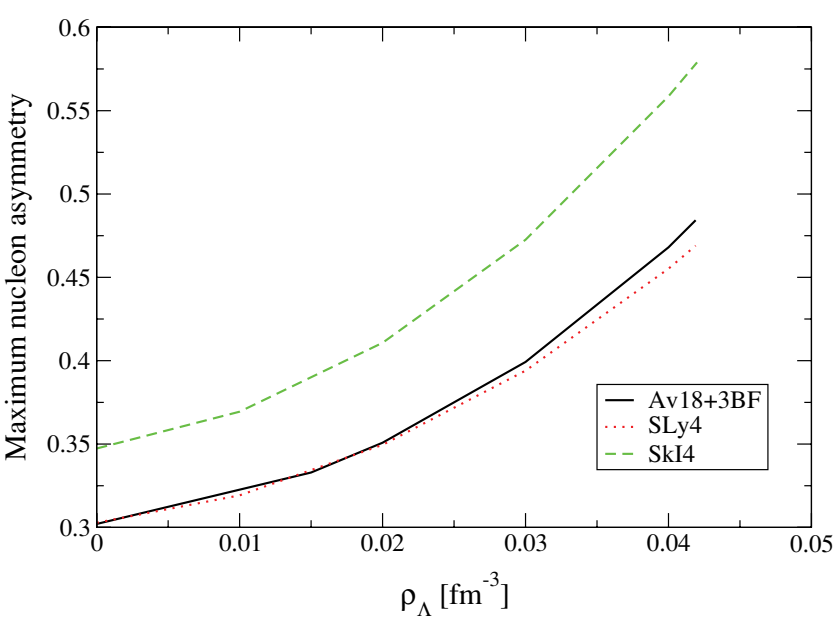

FIG. 3. (Color online) Maximum nucleon asymmetry (neutron drip asymmetry) as a function of $\rho_{\Lambda}$ for three different nucleonic interactions: SLy4 (dotted line), SkI4 (dashed line), and Av18+3BF (solid line).

the paper. The results provided by the Av18+3BF and SLy4 are very similar, while SkI4 produces a slightly larger maximum asymmetry. The relevant fact is that the maximum asymmetry increases with $\rho_{\Lambda}$; this increment at the $\Lambda$ drip point $\rho_{\Lambda} \approx$ $0.04 \mathrm{fm}^{-3}$ is about $18 \%, 16 \%$, and $23 \%$ for Av18+3BF, SLy4, and SkI4, respectively. We remark that qualitatively similar results were obtained in Ref. [3], employing various hyperonic Skyrme forces.

Since in our case all calculations are performed using the same hyperon-nucleon interaction, the differences are due to the different $N N$ interactions. The larger values of the maximum nucleon asymmetry associated to SkI4 can be easily understood by examining the symmetry energy of nuclear matter shown in Fig. 4. In fact in the region of interest, up to $\rho_{N} \approx 0.2 \mathrm{fm}^{-3}$, SLy4 and Av18+3BF provide very similar results for the symmetry energy, which are systematically

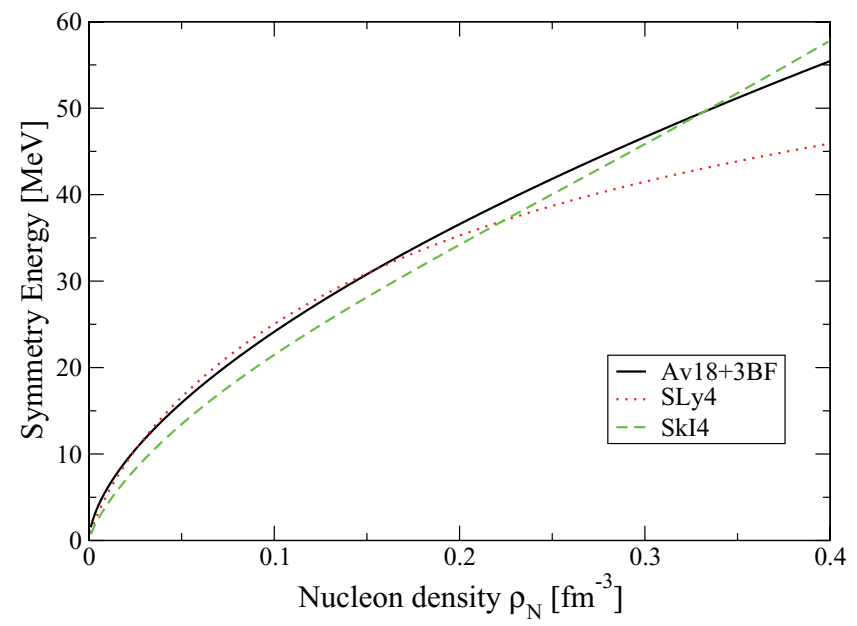

FIG. 4. (Color online) Symmetry energy of nuclear matter for the three different $N N$ interactions used in the paper: SLy4 (dotted line), SkI4 (dashed line), and Av18+3BF (solid line). 
larger than those of SkI4. Therefore the latter interaction facilitates the creation of larger nuclear asymmetry.

It is not easy from these bulk matter results to make quantitative predictions on how the presence of one or more $\Lambda$ 's will affect the neutron drip line in the case of finite nuclei, due to the presence of shell structure, pairing, and deformation in that more complex environment. In any case the effect in homogeneous matter seems sizable enough to motivate explicit calculations with finite nuclei. They will be discussed in the next section.

\section{B. Hypernuclei}

The influence of a few hyperons on the nucleonic structure of a nucleus is usually small: From Fig. 1 we deduce shifts (additional attraction) of the order of $1 \mathrm{MeV}$ for the neutron and proton chemical potentials in the presence of typical $\Lambda$ densities of the order of $0.01 \mathrm{fm}^{-3}$ in a hypernucleus. In order to find appreciable effects we therefore focus on nuclei close to the neutron drip line where the highest (partially) occupied neutron single-particle level is very weakly bound or where additional bound states can be expected with little added attraction, in particular we perform an exploratory study of the neutron-rich isotopes of beryllium and oxygen. In this case the addition of $\Lambda$ 's might stabilize an otherwise unbound neutron level and thus allow the existence of new isotopes or extend the neutron drip point. [By existence we mean the fulfillment of two simultaneous conditions in our model: (i) A solution of the SHF Schrödinger equation with negative single-particle energies for all occupied neutron levels. (ii) This solution lies in a (local) minimum of the energy vs. deformation plot.] Furthermore the lifetime of very short-lived isotopes (neutron emitters beyond the drip line) might be increased (up to the typical hypernucleus lifetime of the order of $100 \mathrm{ps}$ ) and neutron halo features might be either augmented or reduced with respect to the parent nucleus.

In the theoretical treatment the same features might be caused by relaxing the constraint of spherical symmetry and performing deformed SHF calculations instead of spherical ones. It is thus essential to properly take into account this competing effect. In order to remain realistic, we compare in the following ordinary nuclei and double-lambda hypernuclei, which is the maximum that is perhaps experimentally feasible.

We begin in Fig. 5 with the complete chain of Be isotopes and their properties obtained with the SLy4 force, namely the neutron single-particle energies (upper panel), the total binding energies (middle panel), and (lower panel) the quadrupole deformation parameter $\beta_{2}=\sqrt{\frac{\pi}{5}} \frac{\left\langle 2 z^{2}-r^{2}\right\rangle}{\left\langle z^{2}+r^{2}\right\rangle}$ in cylindrical coordinates. With the SLy4 Skyrme force we find beryllium isotopes with $N \leqslant 8$ and $N=10,11,12,14,16$. The neutron drip defined by the minimum of the $B$ vs. $N$ curve lies at ${ }^{12} \mathrm{Be}$, and the heavier isotopes $(N>8)$ are thus unstable with respect to one or two neutron emission. Experimentally the isotopes up to ${ }^{16} \mathrm{Be}$ are known [22] and ${ }^{15} \mathrm{Be}$ and ${ }^{16} \mathrm{Be}$ may decay via neutron emission and are therefore very short-lived. The heaviest isotopes are extremely unstable due to the very small binding energies of the highest occupied neutron single-particle state. (The neutron Fermi energy is indicated by

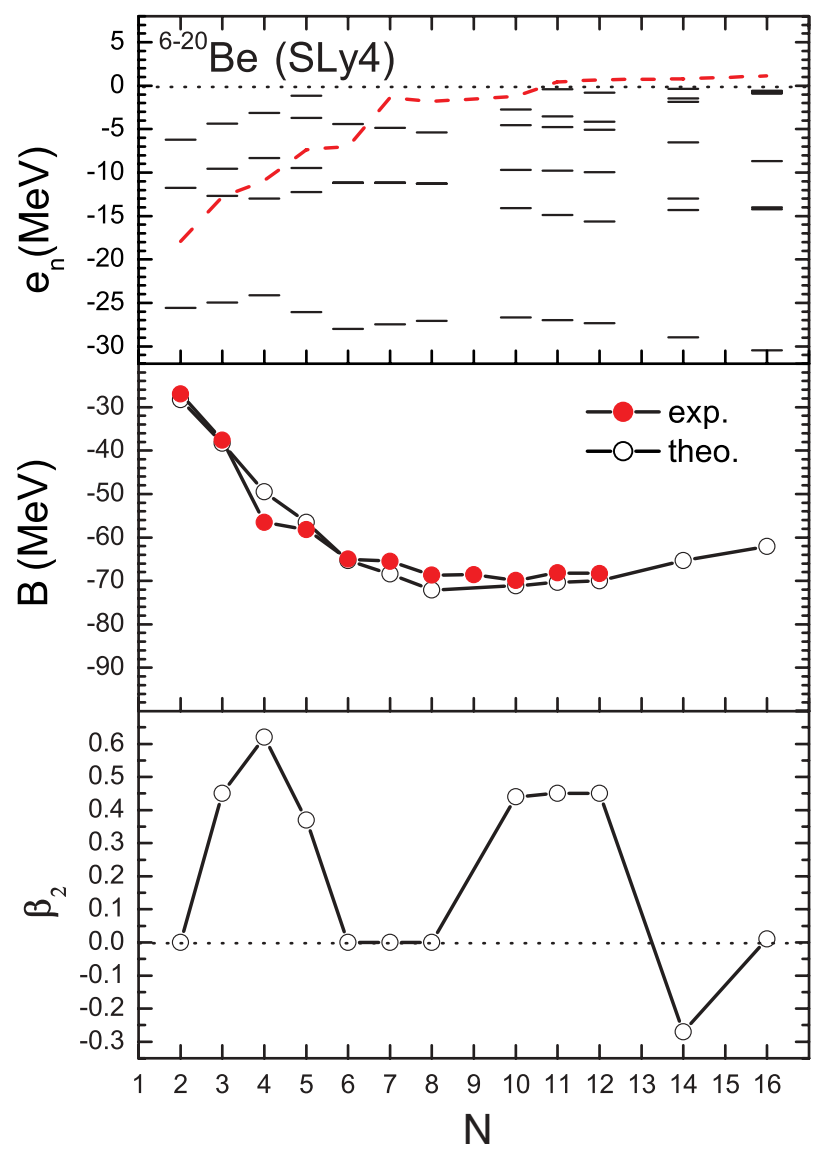

FIG. 5. (Color online) Neutron single-particle levels (upper panel), binding energies (middle panel), and quadrupole deformations (lower panel) of several Be isotopes, obtained with the SLy4 force. The (red) dashed line indicates the neutron Fermi energy.

a red-dashed line in the figure.) We find in fact that the isotopes in the range $8<N<16$ only exist due to the deformation of the nucleus; in spherical calculations the highest neutron level is unbound in these cases. In addition, $N=14$ and $N=16$ are metastable deformed states, i.e., they lie in local minima of the energy vs. deformation plot, whereas in the global minima their highest neutron levels would be unbound. Finally, the odd nuclei $N=9,13,15$ do not exist due to pair breaking.

The plot thus demonstrates the importance and interplay of deformation and pairing for weakly bound isotopes close to the drip line. In order to study these aspects and the effect of added hyperons in more detail, we show in Fig. 6 the energy of the highest (partially) occupied neutron $1 d_{5 / 2}$ single-particle level in several Be isotopes without $\Lambda$ 's and with two $\Lambda$ 's and with or without deformation, obtained using the SLy4 force. We note that in general the addition of $\Lambda$ 's as well as allowing deformation increases clearly the neutron binding energy. More precisely, in the undeformed case without $\Lambda$ 's only the isotopes $N \leqslant 8$ and $N=16$ exist, while the addition of two $\Lambda$ 's stabilizes also $N=14$. With deformation the isotopes $N \leqslant 8$ and $N=10,11,12,14,16$ exist, and the addition of $\Lambda$ 's substantially augments their binding energies and allows also the $N=9,13$ nuclei, overcoming the pair breaking effect. Thus, even if in this case the neutron drip point $N=8$ 


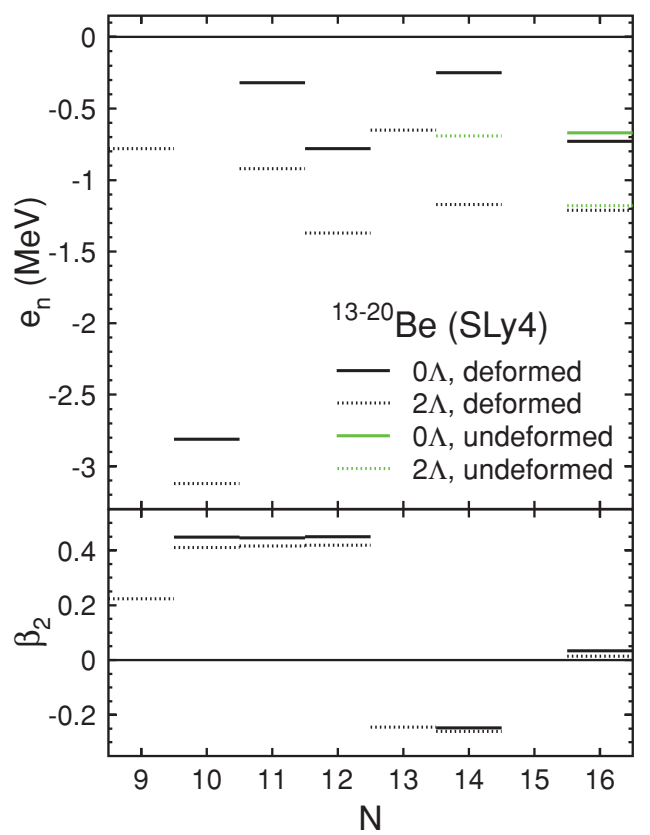

FIG. 6. (Color online) Upper panel: Energy of the highest (partially) occupied neutron $1 d_{5 / 2}$ single-particle level of several beryllium isotopes containing no (solid lines) or two (dotted lines) $\Lambda$ 's. Deformed (black) and undeformed (green) SHF calculations with the SLy4 force are compared. Lower panel: Quadrupole deformation of the (hyper)nucleus.

(minimum of the $B$ vs. $N$ curve for Be) is not shifted, the two short-lived isotopes $N=9,13$ are obtained by the addition of two lambdas.

On the contrary, the same calculations using the SkI4 force yield the neutron drip at $N=8$ and with two $\Lambda$ 's also $N=10$ exists. No other isotopes with $N>8$ are found in this case. This demonstrates the strong dependence of the predictions on the nuclear Skyrme forces used, which are mainly constrained by nuclear data far from the drip line. Nevertheless, the main qualitative effect of added hyperons is clearly demonstrated: nuclei close to the drip line are stabilized and new isotopes are potentially made available.

In Fig. 7 we show the equivalent results for oxygen nuclei. In this case all isotopes up to $N=20$ exist with both Skyrme interactions. The deformations are very small and not shown here. One observes clearly the attractive effect of the two $\Lambda$ 's on the neutrons, even if no new isotopes are made available. The shifts of the neutron single-particle levels are slightly smaller than for Be nuclei, because the $\Lambda$ partial densities are smaller in the larger $\mathrm{O}$ nucleus.

The lower panels of the figure show the one-neutron separation energies computed from the binding energies, $S_{n}=B(N, Z)-B(N-1, Z)$. The difference between $S_{n}$ and $-e_{n}$ is due to the rearrangement of the core (including the change of deformation) of the two nuclei involved. In general the separation energies are slightly smaller in magnitude than the single-particle energies, in particular, $S_{n}$ can become negative while the valence neutrons are still all bound. For our purpose, however, the changes of both quantities due to the addition of two $\Lambda$ 's are very similar and of the order of some hundreds of $\mathrm{keV}$. Comparing with the experimental values (red-dashed lines) one sees that also in this case neither of the two Skyrme forces can give a really satisfactory description of the neutron-rich isotopes. In particular, the theoretical predictions give positive separation energies up to $N=20$, whereas experimentally the neutron drip point is $N=16$ [22,23]. (Note, however, that for $N=17, \ldots, 20$ the "experimental" data involve systematic extrapolations, see Ref. [22].) However, this deficiency is not thought to affect significantly the energy gain due to the addition of two $\Lambda$ 's extracted from the plotted results.

Confronting the results obtained with the two Skyrme forces, one notes that the valence neutrons in the heaviest isotopes $(N>16)$ are slightly more bound with the SkI4 force, whereas the opposite is true for the lighter isotopes. This demonstrates the importance of finite-size effects beyond the indications given by the nuclear matter results, where the SkI4

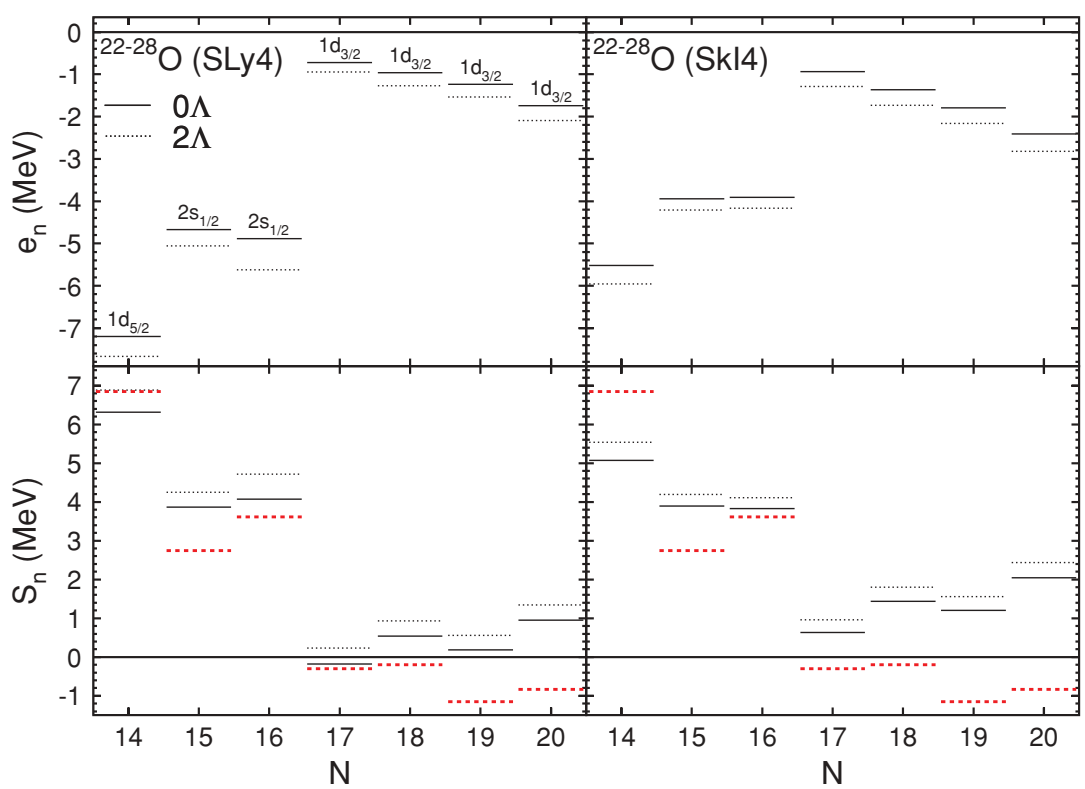

FIG. 7. (Color online) Energy of the highest (partially) occupied neutron single-particle level (upper panels) and one-neutron separation energies (lower panels) of several oxygen isotopes containing no (solid lines) or two (dotted lines) $\Lambda$ 's, using the SLy4 (left panels) and the SkI4 (right panels) nucleonic Skyrme forces. The (red) dashed lines indicate experimental data from Ref. [22]. 
symmetry energy is the smaller one. In particular the spin-orbit parts (and their isospin dependence) of the two Skyrme forces are very different, have no influence on the nuclear matter results, but play an important role in light nuclei.

\section{CONCLUSIONS}

We studied the effect of adding $\Lambda$ hyperons to nuclear matter or finite nuclei, in particular in view of the modification of the neutron drip properties. We used a deformed Hartree-Fock approach with a microscopic in-medium $\Lambda N$ force derived from BHF calculations of hypernuclear matter, together with the modern nucleonic SkI4 or SLy4 Skyrme forces, including nucleonic pairing correlations. The effect is particulary strong in light nuclei due to the relatively high $\Lambda$ partial densities involved, and might stabilize otherwise unbound isotopes, or increase the lifetime of existing ones beyond the neutron drip line. This has been demonstrated explicitly in exploratory calculations of neutron-rich beryllium and oxygen isotopes. Clearly, the quantitative results depend on the $\Lambda N$ force and in particular on the nucleonic force used, which should be refined in the future for these exotic situations in order to allow more precise predictions, possibly within a more sophisticated theoretical framework suited for the delicate problem of paired weakly bound halo states [24].

\section{ACKNOWLEDGMENTS}

Useful discussions with A. Ramos and A. Rios are gratefully acknowledged. This work was supported by the National Science Foundation of China under contract No. 10605018, the Program for New Century Excellent Talents in University under contract No. NCET-07-0730, and Grant Nos. FIS2005-03142 (Ministerio de Educación y Ciencia) and 2005SGR-00343 (Generalitat de Catalunya).
[1] O. Hashimoto and H. Tamura, Prog. Part. Nucl. Phys. 57, 564 (2006).

[2] L. Majling, Nucl. Phys. A585, 211 (1995); H. Tamura, Eur. Phys. J. A 13, 181 (2002).

[3] D. E. Lanskoy and T. Y. Tretyakova, Z. Phys. A 343, 355 (1992).

[4] C. B. Dover and A. Gal, Nucl. Phys. A560, 559c (1993); A. Gal and C. B. Dover, Nucl. Phys. A585, 1c (1995); J. Schaffner, C. B. Dover, A. Gal, C. Greiner, D. J. Millener, and H. Stöcker, Ann. Phys. (NY) 235, 35 (1994); J. SchaffnerBielich and A. Gal, Phys. Rev. C 62, 034311 (2000).

[5] H.-J. Schulze, A. Lejeune, J. Cugnon, M. Baldo, and U. Lombardo, Phys. Lett. B355, 21 (1995); H.-J. Schulze, M. Baldo, U. Lombardo, J. Cugnon, and A. Lejeune, Phys. Rev. C 57, 704 (1998).

[6] I. Vidaña, A. Polls, A. Ramos, M. Hjorth-Jensen, and V. G. J. Stoks, Phys. Rev. C 61, 025802 (2000).

[7] D. Vretenar, W. Pöschl, G. A. Lalazissis, and P. Ring, Phys. Rev. C 57, R1060 (1998).

[8] H. F. Lü, J. Meng, S. Q. Zhang, and S.-G. Zhou, Eur. Phys. J. A 17, 19 (2003).

[9] T. Y. Tretyakova and D. E. Lanskoy, Eur. Phys. J. A 5, 391 (1999).

[10] C. Samanta, P. Roy Chowdhury, and D. N. Basu, J. Phys. G 35, 065101 (2008).

[11] M. Baldo, G. F. Burgio, and H.-J. Schulze, Phys. Rev. C 61, 055801 (2000); H.-J. Schulze, A. Polls, A. Ramos, and I. Vidaña, ibid. 73, 058801 (2006).

[12] P. M. M. Maessen, Th. A. Rijken, and J. J. de Swart, Phys. Rev. C 40, 2226 (1989).
[13] R. B. Wiringa, V. G. J. Stoks, and R. Schiavilla, Phys. Rev. C 51, 38 (1995).

[14] P.-G. Reinhard and H. Flocard, Nucl. Phys. A584, 467 (1995).

[15] E. Chabanat, P. Bonche, P. Haensel, J. Meyer, and R. Schaeffer, Nucl. Phys. A627, 710 (1997); A635, 231 (1998); A643, 441 (1998).

[16] H. Heiselberg and M. Hjorth-Jensen, Phys. Rep. 328, 237 (2000).

[17] A. Akmal, V. R. Pandharipande, and D. G. Ravenhall, Phys. Rev. C 58, 1804 (1998).

[18] J. Cugnon, A. Lejeune, and H.-J. Schulze, Phys. Rev. C 62, 064308 (2000).

[19] I. Vidaña, A. Polls, A. Ramos, and H.-J. Schulze, Phys. Rev. C 64, 044301 (2001).

[20] X. R. Zhou, H.-J. Schulze, H. Sagawa, C. X. Wu, and E. G. Zhao, Phys. Rev. C 76, 034312 (2007).

[21] I. Vidaña, A. Polls, A. Ramos, and M. Hjorth-Jensen, Nucl. Phys. A644, 201 (1998).

[22] G. Audi, O. Bersillon, J. Blachot, and A. H. Wapstra, Nucl. Phys. A729, 3 (2003); A. H. Wapstra, G. Audi, and C. Thibault, ibid. A729, 129 (2003); A729, 337 (2003); IAEA Nuclear Data Services, Atomic Mass Data Center, http://wwwnds.iaea.org/amdc/; BNL National Nuclear Data Center, Chart of Nuclides, http://www.nndc.bnl.gov/chart/.

[23] C. R. Hoffman et al., Phys. Rev. Lett. 100, 152502 (2008).

[24] J. Dobaczewski, W. Nazarewicz, T. R. Werner, J. F. Berger, C. R. Chinn, and J. Dechargé, Phys. Rev. C 53, 2809 (1996); J. Terasaki, S. Q. Zhang, S. G. Zhou, and J. Meng, ibid. 74, 054318 (2006). 